Editorial

\title{
Acknowledgement to Reviewers of Diseases in 2018
}

\section{Diseases Editorial Office}

MDPI, St. Alban-Anlage 66, 4052 Basel, Switzerland

Published: 11 January 2019

Rigorous peer-review is the corner-stone of high-quality academic publishing. The editorial team greatly appreciates the reviewers who contributed their knowledge and expertise to the journal's editorial process over the past 12 months. In 2018, a total of 107 papers were published in the journal, with a median time to first decision of 14 days and a median time to publication of 33 days. The editors would like to express their sincere gratitude to the following reviewers for their cooperation and dedication in 2018:

\begin{tabular}{ll} 
Aasvang, Gunn Marit & Carneiro, Sueli Coelho Da Silva \\
Addington, Caroline & Castell Escuer, Margarida \\
Ahlenstiel, Chantelle & Cauli, Omar \\
Alexandre, Mezentsev & Cavé, Christian \\
Allen, Herbert & Cécile, Oury \\
Antoñanzas, Fernando & Chalah, Moussa \\
Arndt, Patrick G. & Chamcheu, Jean Christopher \\
Athyros, Vasilios & Chang, Chien-Chung \\
Bachanova, Veronika & Chaudhary, Ujwal \\
Bakillah, Ahmed & Chen, Wenchun \\
Bakonyi, Tamás & Chen, Yann-Jang \\
Balan, Murugabaskar & Chendrasekhar, Akella \\
Bandea, Claudiu & Chien, Yin-Hsiu \\
Baragetti, Andrea & Cicero, Arrigo Francesco Giuseppe \\
Basini, Giuseppina & Cimmino, Giovanni \\
Bates, John T. & Clement, Sophie \\
Battino, Maurizio & Correia Santos, Nadine \\
Battistelli, Cecilia & Coussens, Scott \\
Beckman, Joan & Craig-Kuhn, Megan Clare \\
Bertrand, Gérald & Cruz-Sosa, F. \\
Bodet, Charles & Cullen, Paul \\
Borgstahl, Gloria E. O. & Danesh-Sani, Seyed Amir \\
Bortkiewicz, Alicja & Dawidowicz, A. L. \\
Boyanova, Lyudmila & De Berardis, Domenico \\
Brameld, John & De Geest, Bart \\
Brooke, Joanne & De Pietro, Giuseppe \\
Budzi, Doroth & Deevska, Gergana \\
Buigues, Cristina & Dehghannasiri, Roozbeh \\
Capasso, Anna & Desselberger, Ulrich \\
& \\
\hline &
\end{tabular}


Devadas, Krishnakumar

Di Fazio, Pietro

Di Martino, Giuseppe

Dibaba, Daniel

Dimopoulos, Konstantinos

Ding, Yinyuan

Domingo-Gonzalez, Racquel

Dosoky, Noura

Dou, Xiaoguang

Downer, Brian

Du, Xiao-jun

Dubińska-Magiera, Magda

Edwards, Erika

Eilertsen, Karl-Erik

Espuny, Antoni Camins

Ezhov, Marat V.

Fernandez, Maria Luz

Fetissov, Sergueï O.

Franconi, Rosella

Frisher, Martin

Fukuda, Yoshiharu

Furuhashi, Kazuki

Gaddam, Ravinder Reddy

Garus-Pakowska, Anna

Ghareh Baghi, Arash

Goroll, Allan H.

Gorska-Ponikowska, Magdalena

Gray, Andrew

Green, James A.

Greenberger, Joel S.

Guido, Luis F.

Gyawali, Pradip

Hanai, Jun-ichi

Harvey, Adam

Hassan, Amany

Head, Michael

Hegde, Muralidhar L.

Heggermont, Ward

Higa, Gerald M.

Hingorani, Dina

Hioki, Hirofumi

Hodge, Allison

Hodgson, Ian
Hoffman, Richard

Huang, Guan-Jhong

Hubková, Beáta

Hung, Li-Fang

Ichikawa, Hiroshi

Italia, Salvatore

Ivanov, Alexander V

Jacova, Claudia

Jeckel, Kimberly M

Jerebtsova, Marina

Johnston, Simon

Jordão, António Manuel

Just, Nathalie

Kabała-Dzik, Agata

Kakisis, John D.

Kam, Yiu Wing

Kang, Sinyoung

Kang, Jae Seung

Karanis, Panagiotis

Karkhanis, Vrajesh

Karlin, Bradley

Keightley, Cristina

Kim, Kyung-su

Kim, Hyung Sik

Kojima, Masami

Kones, Richard

Konstandin, Mathias

Konstantinidou, Valentini

Kosmas, Constantine E.

Koyama, Shin

Krajewska-Włodarczyk, Magdalena

Kujawska, Małgorzata

Kumar, Binod

Kumar, Mukesh

Kurdowska, Anna K.

Kwan, Hiu-yee

Lang, Chim

Langhammer, Birgitta

Lass, Richard

Lauschke, Volker

Lava, Sebastiano A.G.

Lebaron, Richard

Ledda, Caterina 
Ledesma, Maria Dolores

Lee, Gihyun

Leibowitz, Avshalom

Leimanis, Mara

Li, Zijian

Liang, Yan

Lin, Gen-Min

Lin, Huey-Jen

Lin, Chih-Li

Lin, Liang-Tzung

Lin, Ro-Ting

Liu, Wei

Liu, Pei-Yang

Liu, Yu

Lombardi, Raffaella

Lonardo, Amedeo

Londono, Berlin

Losurdo, Giuseppe

Lozano, Jose Manuel

Lu, Zhanping

Lu, Yufeng

Luca, Maria

Luca, Adrian

Lund, Elizabeth K.

Maclellan, Reid A.

Madeira De Carvalho, Luis Manuel

Maeder, Micha Tobias

Maher, Pamela

Maiorano, Eugenio

Malfeito Ferreira, Manuel

Mammadova-Bach, Elmina

Mammola, Caterina Loredana

Marengo, Barbara

Marlicz, Wojciech

Martínez Álvarez, José Ascención

Martini, Maurizio

Masalova, Olga V.

Matouskova, Petra

Matsuo, Muneaki

Matsuura, Bunzo

McBride, Devin W.

McCarty, Catherine

McCormack, Joseph G.
McCown, William

Mei, Ya-Fang

Mendez-Sanchez, Nahun

Meschini, Stefania

Mesquita, João

Metwally, Mayada

Metz, Stefan W.

Milavetz, Barry

Miranda, Cristobal

Mitra, Amal K.

Mlocicki, Daniel

Monroy, Fernando

Moreno, Margarita

Mou, Haiwei

Mukaetova-Ladinska, Elizabeta B

Mukhopadhyay, Tuli

Muto, Akira

Nanno, Masanobu

Naruishi, Koji

Navarro-Martínez, Rut

Nemeth, Lynne

Nifli, Artemissia-Phoebe

Nissapatorn, Veeranoot

Nodomi, Seishiro

Obeng-Gyasi, Emmanuel

Olivier, Jean Luc

Omar, Syed Haris

Onyenwoke, Rob U

Oon, Hazel H.

Ortolano, Saida

Perez Gregorio, Maria Rosa

Pérez-Prieto, Daniel

Perry, Rachel

Peterson, Julia

Piche, Marie-Eve

Pinent, Montserrat

Pistelli, Francesco

Plaza-Díaz, Julio

Pokorn, Marko

Posada-Quintero, Hugo

Povey, Andrew

Probst, Yasmine

Procházka, Aleš 
Prokai, Laszlo

Ragusa, Andrea

Rahman, Muhammad Aziz

Rajamani, Sathish

Ranzato, Elia

Raposio, Edoardo

Rather, Irfan Ahmad

Rathore, Abhay PS

Reid, St Patrick

Ren, Zefang

Reyes-Sandoval, Arturo

Reynoso, Elena

Risbano, Michael

Rodenbeck, Stacey Dineen

Rossi, Miriam

Rosso, Natalia

Rozsival, Pavel

Rudolph, Bryan

Saera-Vila, Alfonso

Salvati, Bruno

Santulli, Gaetano

Saraux, Alain

Sassi, Yassine

Satake, Hironaga

Sato, Marcello Otake

Seeman, Mary V.

Shepherd, Samantha

Sherer, Nathan

Sherwin, LeeAnne B.

Shi, Jiaqi

Shieh, Tzong-Ming

Shim, Joon W.

Shipman, Kate

Si, Dong

Siniscalco, Dario

Smith, Barry C.

Song, Byeongwoon

Song, Chunhua

Sonntag, Kai

Soudeyns, Hugo

Spassov, Sashko

Stahelin, Robert

Stauber, Rudolf E.
Stefani, Laura

Subburaju, Sivan

Sun, Xiaoming

Tai, Wanbo

Tan, BK

Tarantino, Giovanni

Teng, Ba-Bie

Teschke, Rolf

Toribio-Mateas, Miguel

Torquati, Luciana

Tovoli, Francesco

Trovato, Guglielmo

Turiel, Maurizio

Ufnal, Marcin

Unchwaniwala, Nuruddin

Urbanelli, Lorena

Van Der Spoel, Aarnoud C.

Van Hoek, Bart

Van Zanten, Malou

Varrica, Daniela

Vautier, Simon

Velez, Zélia

Venkatesh, Siddarth

Veraldi, Stefano

Vergara, Daniele

Vinciguerra, Manlio

Vitetta, Luis

Vogtmann, Emily

Vozza, Iole

Wada, Jun

Wall-Medrano, Abraham

Walther, Wolfgang

Wang, Zeneng

Wang, Xiaoqin

Weinstein, Philip

Weir, Dawn

Wenner, Yaroslava

Woo, So-Youn

Xiang, Yu

Yasuda, Kazuki

Yehuda, Shoenfeld

Yi, Tao

Yiin, Lih-Ming 
Yu, Chia-Li

\section{Zhu, Qiuyu Martin}

Zhang, Yu

(C) 2019 by the authors. Licensee MDPI, Basel, Switzerland. This article is an open access article distributed under the terms and conditions of the Creative Commons Attribution (CC BY) license (http://creativecommons.org/licenses/by/4.0/). 